\title{
Generation of Second-Harmonic Conical Waves via Nonlinear Bragg Diffraction
}

\author{
Solomon M. Saltiel, ${ }^{1,2}$ Dragomir N. Neshev, ${ }^{1}$ Robert Fischer, ${ }^{1}$ Wieslaw Krolikowski, ${ }^{1}$ Ady Arie, ${ }^{3}$ and Yuri S. Kivshar ${ }^{1}$ \\ ${ }^{1}$ Nonlinear Physics Center and Laser Physics Center, Center for Ultra-high Bandwidth Devices for Optical Systems (CUDOS), \\ Research School of Physical Sciences and Engineering, Australian National University, Canberra ACT 0200, Australia \\ ${ }^{2}$ Department of Quantum Electronics, Faculty of Physics, Sofia University, Bulgaria \\ ${ }^{3}$ School of Electrical Engineering, Faculty of Engineering, Tel-Aviv University, Tel-Aviv, Israel
}

(Received 3 August 2007; published 12 March 2008)

\begin{abstract}
We report on the observation of second-harmonic conical waves generated in a novel geometry of the transverse excitation of an annular periodically poled nonlinear photonic structure by a fundamental Gaussian beam. We show that the conical beams are formed as a result of the higher-order nonlinear Bragg diffraction involving two parametric processes in which an ordinary fundamental wave is converted simultaneously into ordinary and extraordinary polarized second harmonics.
\end{abstract}

PACS numbers: 42.65.Ky, 42.25.Fx, 42.70.Qs

The process of the second-harmonic generation (SHG), i.e., conversion of two photons of the fundamental wave into a single photon at the doubled frequency, is one of the most intensively studied effects in nonlinear optics [1]. It is well established that the efficiency of the second-order and other similar nonlinear processes depends crucially on the phase-matching relations between the interacting waves. In birefringent optical crystals these conditions are achieved by employing either temperature or angle tuning. In nonlinear optical media with weak birefringence an efficient frequency conversion can be achieved by using the quasiphase-matching (QPM) technique [2,3] that involves a periodic modulation of the second-order nonlinearity of the material. In the ferroelectric crystals such as $\mathrm{LiNbO}_{3}$ or $\mathrm{LiTaO}_{3}$, the QPM conditions can be easily realized by spatial periodic poling [4]. In this way, both one- and two-dimensional nonlinear periodically modulated photonic structures can be fabricated, and they are often called $\chi^{(2)}$ (or quadratic) photonic crystals [5,6]. Depending on the symmetry and geometry of the poling pattern, the efficient SHG can be realized for the waves propagating in particular spatial directions [7]. The typical geometries involve, in one spatial dimension, single- or multiperiod rectangular ferroelectric pattern, and in two spatial dimensions, planar structures with different symmetries (square, rectangular, hexagonal, centered rectangular, and oblique).

In this Letter, we report on the first realization of the second-harmonic interactions in a novel transverse geometry and on the generation of second-harmonic ( $\mathrm{SH}$ ) conical waves. We employ a new type two-dimensional nonlinear photonic crystal with an annular periodically poled QPM structure, and demonstrate experimentally that $\mathrm{SH}$ waves acquire a conical wave structure so that the corresponding SH rings represent the far field of the Bessel beams generated inside the nonlinear crystal via multiple order nonlinear Bragg diffraction.

We consider a two-dimensional nonlinear photonic structure with a circular periodic array of ferroelectric domains and a constant linear refractive index [8]. In

general, such two-dimensional QPM photonic structures have been explored exclusively in the longitudinal geometry of the SHG when the fundamental beam propagates perpendicularly to the domains boundaries of periodically varying second-order nonlinearity $[5,6,8,9]$. Here, we explore a novel transverse geometry when the fundamental light beam propagates along the axis of the structure. The front facet of the annular periodically poled stoichiometric lithium tantalate (SLT) sample used in our experiments with clearly visible domain boundaries is shown in Fig. 1(a) [for technical details, see Ref. [8] ]. It is a $Z$ cut, $L=0.49 \mathrm{~mm}$ thick slab with the QPM period of $7.5 \mu \mathrm{m}$ and duty factor varying inside the sample from 0.7 at $(Z+)$

(a)

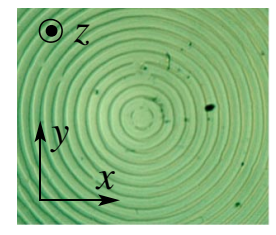

(b)

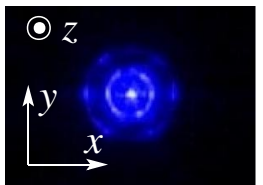

(d)
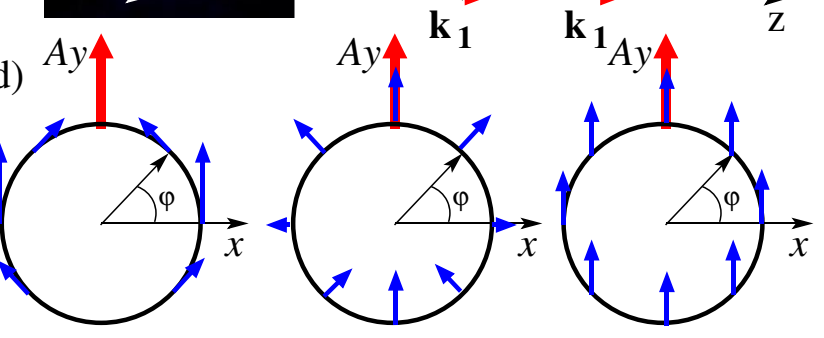

FIG. 1 (color online). (a) Front facet of the SLT sample. (b) Far-field image of first two diffraction SH rings. (c) Phasematching diagram of the SHG process in the transverse QPM grating. (d) Polarization structure of the ordinary (left), extraordinary (middle), and small-angle (right) SH rings. The fundamental wave (red or gray) is linearly (along $Y$ ) polarized. 
surface to 0.8 at $(Z-)$ surface. Both, $(Z+)$ and $(Z-)$ surfaces are polished. Small $20 \mathrm{~nm}$ deep grooves that remain after polishing cause weak (less than 3\%) diffraction of the fundamental beam which we send perpendicularly to the domain structure, along its symmetry axis. As a light source we use a Ti:sapphire oscillator and regenerative amplifier delivering beam of 140 fs pulses, with a repetition rate of $250 \mathrm{kHz}$ and average output power of $740 \mathrm{~mW}$.

The input beam is loosely focused such that its waist diameter at the input facet of the sample is $147 \mu \mathrm{m}$ (FWHM) which, for a given input power, corresponds to an intensity of about $100 \mathrm{GW} / \mathrm{cm}^{2}$. The beam covers roughly 10 domain rings. We observe that the transverse illumination of the radial structure by the fundamental beam leads to multiple conical emission of the $\mathrm{SH}$ waves [see Figs. 1(b) and 2(a)]. These waves form a set of rings in the far field, and their propagation angles satisfy exact Bragg relation, i.e., the cone angles, as has been verified in the experiment, are determined by the ratio of the wavelength of the second harmonic to the period of the modulation of the $\chi^{(2)}$ nonlinearity.

To explain why the SH rings appear, we consider the phase-matching conditions [10] for the corresponding parametric processes shown in Fig. 1(c). The general vectorial phase-matching condition

$$
\mathbf{2} \mathbf{k}_{1}+\mathbf{G}_{m}+\Delta \mathbf{k}_{\mathbf{m}}=\mathbf{k}_{2}^{(\mathbf{m})},
$$

(with $\mathbf{k}_{1}$ and $\mathbf{k}_{2}$ wave vectors of the fundamental and $\mathrm{SH}$ waves, $\mathbf{G}_{m}$ the $m$ order of reciprocal vector of the $\chi^{(2)}$ modulation $G_{m}=m(2 \pi / \Lambda), \Delta k_{m}$ the mismatch, and $m$ the order of the generated SH wave) will be analyzed by splitting into the transverse and the longitudinal phasematching conditions [11]. For our case the two conditions will be: the transverse ones, $k_{2} \sin \alpha_{m}=G_{m}=m G_{1}$, and the longitudinal ones, $k_{2} \cos \alpha_{m}-2 k_{1}=\Delta k_{m}$. With a correct choice of the fundamental wavelength or temperature, a given diffraction order will be longitudinally phase
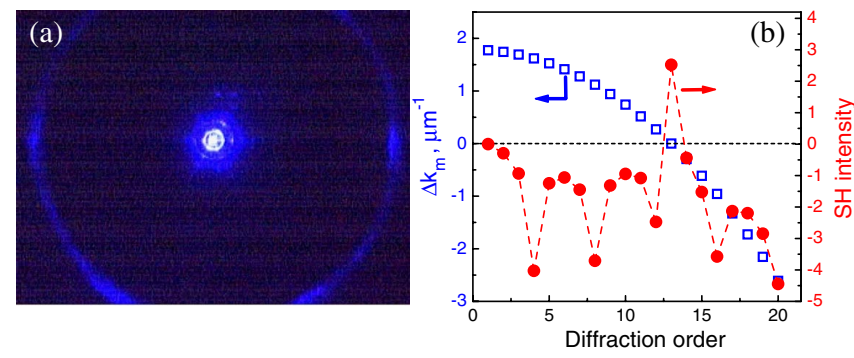

FIG. 2 (color online). Far-field image of experimentally observed $\mathrm{SH}$ rings. The axis $X$ is horizontal. (a) The fundamental beam is exactly in the center of the annular structure: first several orders and 13th order SH ring are seen. (b) Dependencies of the wave vector mismatch and the predicted SH efficiency normalized to the first-order SH efficiency (in log scale) as a function of the diffraction order. matched $\left(\Delta k_{m}=0\right)$. In the example shown in Fig. 1(c) the third diffraction order is phase matched $\left(\Delta k_{3}=0\right)$, and for this interaction both the phase-matching conditions are satisfied simultaneously. Inside the crystal, the propagation angles of all transversely phase-matched waves can be found from the relation $\sin \alpha_{m}=m \lambda_{2} / n_{2} \Lambda$, (where $n_{2}=$ 2.31 is the SH index of refraction) which is analogous to the Bragg condition governing the diffraction of waves on periodic modulation of the index of refraction. Here the refractive index is constant but the nonlinear properties of the medium are radially periodic. This effect is known as nonlinear Bragg diffraction, and it was first discussed by Freund in 1968 [12].

The SHG process in the transverse direction $\left(\alpha_{m}=90^{\circ}\right)$ determines the total maximum diffraction orders, $M_{2}=$ $n_{2} \Lambda / \lambda_{2}$. For this particular case the longitudinal mismatch is $\Delta k_{m}=2 k_{1}$, and its compensation is possible only for two counterpropagating fundamental waves [13].

The discussion so far considered the phase-matching conditions in a single plane (single azimuthal angle $\varphi$ defined in Fig. 1). Since the excitation is in the center of the structure the same conclusions will be valid for any azimuthal angle $\varphi$ and consequently the $\mathrm{SH}$ radiation will appear in the form of concentric cones with cone angles $\alpha_{m}$.

The outer $\mathrm{SH}$ rings possess interesting polarization properties determined by the structure of the $\chi^{(2)}$ tensor of the lithium tantalate. This crystal belongs to the $3 \mathrm{~m}$ symmetry point group. For the fundamental beam propagating along the $Z$ axis, the following nonzero components are relevant: $d_{z x x}=d_{z y y}, d_{y x x}=d_{x y x}=-d_{y y y}$. Note that in periodically poled crystals with $3 m$ symmetry the coefficients $d_{x x y}, d_{y x x}, d_{y y y}$ also change signs periodically [14]. Then two types of the SHG processes are possible: type I [e-oo], two ordinary waves generate an extraordinary $\mathrm{SH}$ and type 0 [o-oo], two ordinary waves generate an ordinary SH. We can show that for these two parametric processes the effective nonlinearities can be written in the following form:

$$
\begin{gathered}
d_{\mathrm{eff}}^{(o)}=d_{y y y} \cos (\varphi+2 \gamma), \\
d_{\mathrm{eff}}^{(e)}=d_{y y y} \cos \alpha \sin (\varphi+2 \gamma)+d_{z y y} \sin \alpha,
\end{gathered}
$$

where $\varphi$ is the azimuthal angle measured counterclockwise from the $X$ axis, $\alpha$ is the diffraction angle inside the crystal, and $\gamma$ is the azimuthal angle that defines the polarization of the input beam ( $\gamma=0$ for polarization along $X$ axis). As a result, the polarization of the "ordinary" conical wave is azimuthal [Fig. 1(d) left graph], while the polarization of "extraordinary" conical wave is radial [Fig. 1(d) middle graph].

For small angles $\alpha$ (when $\tan \alpha \ll 1$ ), the difference in the refractive indices of the ordinary and extraordinary waves can be neglected, the ordinary and extraordinary $\mathrm{SH}$ rings perfectly overlap, and as a result the intensity of 
conically emitted light does not depend on $\gamma$ or $\varphi$. In addition, its polarization becomes linear [Fig. 1(d) (right)]. For $\gamma=0$ and for $\gamma=\pi / 2$ polarization of the SH wave coincides with the $Y$ direction (with relevant nonlinear components $d_{y x x}$ and $d_{y y y}$, respectively), while for $\gamma=$ $\pi / 4$ polarization of the $\mathrm{SH}$ wave coincides with the $X$ direction (with the nonlinear component $d_{x y x}$ ).

For relatively large $\alpha$, the ordinary and extraordinary conical waves propagate with different phase velocities $\left[n_{o}>n_{e}(\alpha)\right]$ and at slightly different conical angles. In this case, the output polarization depends strongly on the actual spatial overlap of the two rings since they are orthogonally polarized. In Fig. 1(d) (left and middle) we show the structure of the two predicted polarization components of the ring for $d_{y y y} / d_{z y y} \approx-1.7$ [15] and $\alpha=$ $20^{\circ}$. For relatively long crystals every diffraction order should appear as a doublet consisting of two orthogonally polarized rings.

The azimuthal intensity distributions of the ordinary and extraordinary $\mathrm{SH}$ rings are proportional to $\left[d_{\mathrm{eff}}^{(o)}\right]^{2}$ and $\left[d_{\mathrm{eff}}^{(e)}\right]^{2}$, respectively, and hence depend on the input polarization defined by the angle $\gamma$. Since the two rings are orthogonally polarized the total intensity is given as

$$
I_{2 \omega, m} \propto\left\{S^{(e)}\left[d_{\mathrm{eff}}^{(e)}\right]^{2}+S^{(o)}\left[d_{\mathrm{eff}}^{(o)}\right]^{2}\right\} I_{1}^{2} g_{m}^{2} L^{2},
$$

where $S^{(o, e)}=\sin ^{2}\left(\pi L / 2 L_{\mathrm{coh}}^{(o, e)}\right) /\left(\pi L / 2 L_{\mathrm{coh}}^{(o, e)}\right)^{2}, \quad L_{\mathrm{coh}}^{(o, e)}=$ $\pi / \Delta k_{m}^{(o, e)}$, and $g_{m}=2 /(\pi m) \sin (\pi m D)$ with $D$ being the duty factor. In Fig. 2(b) we illustrate the dependence of the relative intensity $I_{2 \omega, m}^{(o)}$ (in log scale) in each diffraction order $m$ assuming for simplicity that the length of the sample is a multiple of the coherence length $\left(L_{\mathrm{coh}}\right)$.

The wave vectors of SH waves emitted by the annular structure lay on a cone with a conical angle $\alpha$. It is known that a superposition of an infinite number of plane waves with the wave vectors laying on a cone results in a formation of a Bessel beam [16,17]. We have recently shown that a simple annular QPM structure in $\chi^{(2)}$ crystals with $4 \mathrm{~mm}$ symmetry leads to the generation of the Bessel SH beam with a radial polarization [10]. The situation is drastically different in case of media with lower symmetries due to contribution of several components of the $\chi^{(2)}$ tensor to the generated SH. Detailed analysis shows that in media such as $\mathrm{LiTaO}_{3}$ or $\mathrm{LiNbO}_{3}$, the spatial structure of the SH beam inside the crystal has a form of a superposition of the low-order Bessel beams with azimuthally modulated intensity. The exact formulas are quite cumbersome (and will be discussed elsewhere), but they can be simplified in the case of small emission angles $(\tan \alpha \ll 1)$ leading to

$$
E(\rho, \varphi, z) \propto 2 \pi A^{2} d_{y y y} z J_{0}(\xi)\left[\hat{u}_{x} \sin 2 \gamma+\hat{u}_{y} \cos 2 \gamma\right],
$$

where $0<z<L, \xi=k_{2} \rho \sin \alpha$ and $\rho=\left(x^{2}+y^{2}\right)^{1 / 2}$ is the transverse radial coordinate. $\hat{u}_{x}$ and $\hat{u}_{y}$ are unit vectors along $X$ and $Y$. It is clear that the beam represented by Eq. (5) is linearly polarized, with its intensity being independent of the azimuthal coordinate $I \propto 4 \pi^{2} d_{y y y}^{2} A^{4} J_{0}^{2}(\xi)$. This result is in a full agreement with the recorded SH farfield $\varphi$ dependence [Fig. 4(a)].

The observed SH rings for the fundamental wavelength of $822 \mathrm{~nm}$ are shown in Fig. 2(a). The measured conical angles agree well with those determined from the nonlinear Bragg diffraction formula. By rescaling the size of the lower-order diffraction pattern we found that the phasematched SH emission which is represented in the far zone by the ring with the largest diameter and conical angle of $45.6^{\circ}$ corresponds to the high 13 th diffraction order. In Fig. 2(b) we depict the calculated (using the published refractive index data [18]) longitudinal phase mismatch and relative power of the SH waves for $\lambda=836 \mathrm{~nm}$. The highest peak corresponds to the phase-matched $\left(\Delta k_{13}=0\right)$ 13th order SH ring.

The phase-matching curve obtained by scanning the input wavelength around $822.2 \mathrm{~nm}$ is shown in Fig. 3(a) together with the theoretical prediction based on the model from Ref. [10]. We observe good qualitative agreement between the experimental curve and the theoretical prediction. The difference in the phase-matching wavelength ( $822.2 \mathrm{~nm}$, experimental, and $836 \mathrm{~nm}$, theoretical) is most likely due to uncertainty in the value of the ordinary refractive index caused by the lack of accurate corresponding Sellmeier coefficients. In Fig. 3(b) we verify a quadratic dependence of the SH power of the phase-matched 13th order SH ring as a function of the input power. From this figure (obtained at $640 \mathrm{~mW}$ of the fundamental average power taking into account all losses), we obtain the internal efficiency for the phase-matched emission of $\eta_{13}=$ $0.0056 \% / W$. Such low efficiency is attributed to the fact that we use a high order QPM interaction. Additionally, the variations of the duty factor inside the crystal leads to an effective length 2-3 times less than the real sample thickness. The measured efficiency of the SH power generated in the first-order ring is 220 times less than $\eta_{13}$. Our estimation shows that for a $1 \mathrm{~mm}$-long sample operating in the regime of the first-order QPM with homogeneous
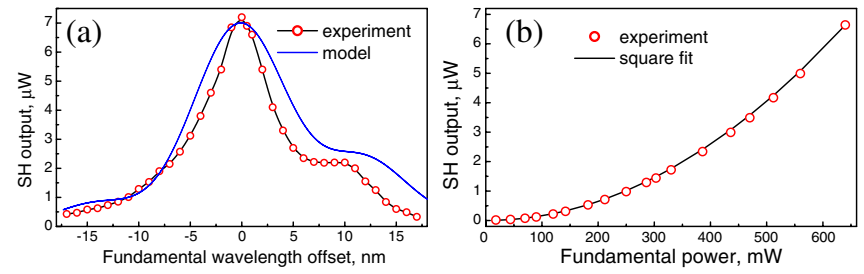

FIG. 3 (color online). (a) Measured (circles) phase-matching curve and theoretically predicted (solid line) phase-matching curve (normalized to the maximum value of the signal obtained at $\lambda=822.5 \mathrm{~nm}$ ). (b) Illustrating quadratic dependence of the 13th order $\mathrm{SH}$ ring on the input power. 

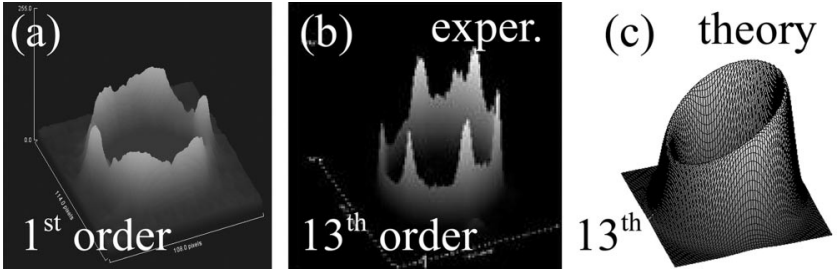

FIG. 4. (a),(b) Experimentally recorded intensity distribution of $m=1$ and $m=13 \mathrm{SH}$ rings. (c) Theoretical prediction for the azimuthal intensity distribution of the $m=13 \mathrm{SH}$ ring evaluated from Eq. (4).

$50 \%$ duty factor a $10 \%$ efficiency of the SH generation could be achieved.

Figures 4(a) and 4(b) show the experimental azimuthal intensity distribution of the 1st and 13th order SH rings. The theoretical prediction for the 13th order $\mathrm{SH}$ ring is given by Eq. (4), and it is shown in Fig. 4(c).

As follows from Fig. 1(b), 4(a), and 4(b), the SH rings exhibit an additional azimuthal modulation with 6 well defined peaks. These peaks are insensitive to the input polarization and intensity, and they appear even in the regime of linear diffraction of the fundamental beam on the $20 \mathrm{~nm}$ deep surface relief - replica of poling pattern. The peaks are artifacts of the discrete character of domain boundaries resulting from the well-known tendency of ferroelectric domains in the SLT to retain their hexagonal shape $[8,19]$.

In Fig. 5 we show our results of the polarization measurements of the SH rings. For the linearly polarized input fundamental beam the generated wave is also linearly polarized in the case of the first-order ring. This result agrees with Eqs. (2) and (3) in the limit $\alpha \rightarrow 0$. The polarization state becomes more complex for higher-order nonlinear diffraction as the output signal contains both ordinary and extraordinary contributions.

In conclusion, we have reported on the first observation of second-harmonic conical waves generated by twodimensional annular nonlinear photonic structures. We have explained the effects observed in experiments by employing the concept of nonlinear Bragg diffraction com-
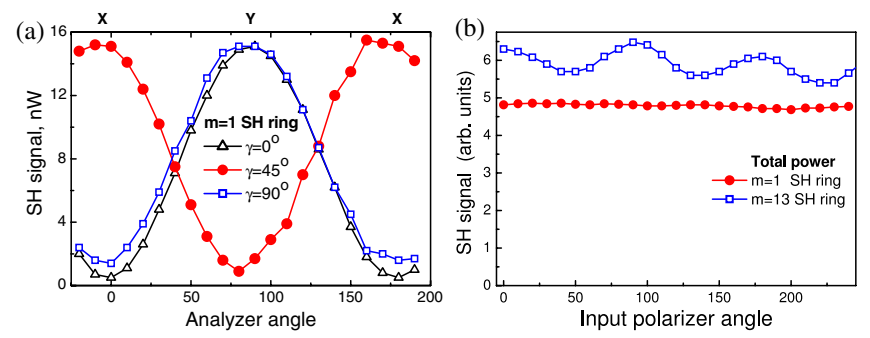

FIG. 5 (color online). Polarization properties of the SH rings: (a) Power of the 1st order SH ring vs analyzer angle, (b) Total power of the 1st and 13th order SH rings vs the input polarization angle. bined with the longitudinal and transverse phase-matching conditions. We have studied the polarization properties of the conical second-harmonic radiation and have described how the beam polarization depends on the crystal symmetry and the conical angle. The geometry studied here may enable one to generate conical harmonics in other systems including, for instance, acoustic waves in dielectrics and matter waves in optical lattices.

This work was supported by the Australian Research Council and the Israeli Science Foundation (Grant No. 960/05). We thank D. Kasimov, A. Bruner, P. Shaier, and D. Eger for their assistance in the fabrication of the SLT samples. S. Saltiel thanks the Nonlinear Physics Center in Australia for hospitality and support.

[1] P. A. Franken and J.F. Ward, Rev. Mod. Phys. 35, 23 (1963).

[2] J. A. Armstrong, N. Bloembergen, J. Ducuing, and P. S. Pershan, Phys. Rev. 127, 1918 (1962).

[3] M. M. Fejer, G. A. Magel, D.H. Jundt, and R. L. Byer, IEEE J. Quantum Electron. 28, 2631 (1992).

[4] M. Houe and P.D. Townsend, J. Phys. D 28, 1747 (1995).

[5] V. Berger, Phys. Rev. Lett. 81, 4136 (1998).

[6] P. Xu, S. H. Ji, S. N. Zhu, X. Q. Yu, J. Sun, H. T. Wang, J.L. He, Y. Y. Zhu, and N. B. Ming, Phys. Rev. Lett. 93, 133904 (2004).

[7] J. R. Kurz, A. M. Schober, D. S. Hum, A. J. Saltzman, and M. M. Fejer, IEEE J. Sel. Top. Quantum Electron. 8, 660 (2002).

[8] D. Kasimov, A. Arie, E. Winebrand, G. Rosenman, A. Bruner, P. Shaier, and D. Eger, Opt. Express 14, 9371 (2006).

[9] N. G. R. Broderick, G. W. Ross, H.L. Offerhaus, D. J. Richardson, and D. C. Hanna, Phys. Rev. Lett. 84, 4345 (2000).

[10] S. Saltiel, W. Krolikowski, D. Neshev, and Yu. S. Kivshar, Opt. Express 15, 4132 (2007).

[11] S. P. Tewari, H. Huang, and R. W. Boyd, Phys. Rev. A 54, 2314 (1996).

[12] I. Freund, Phys. Rev. Lett. 21, 1404 (1968).

[13] R. Fischer, D. N. Neshev, S. M. Saltiel, A. A. Sukhorukov, W. Krolikowski, and Yu. S. Kivshar, Appl. Phys. Lett. 91, 031104 (2007).

[14] A. Ganany, A. Arie, and S. M. Saltiel, Appl. Phys. B 85, 97 (2006).

[15] F. Charra and G. G. Gurzadyan, in Nonlinear Dielectric Susceptibilities, edited by D. F. Nelson (Springer, Berlin, 2000).

[16] J. Durnin, J. Opt. Soc. Am. A 4, 651 (1987).

[17] Z. Bouchal and M. Olivik, J. Mod. Opt. 42, 1555 (1995).

[18] M. Nakamura, Sh. Higuchi, Sh. Takekawa, K. Terabe, Ya. Furukawa, and K. Kitamura, Jpn. J. Appl. Phys. 41, L465 (2002).

[19] V. Shur, E. Shishkin, I. Baturin, A. Chernykh, D. Kuznetsov, A. Lobov, A. Shur, M. Dolbilov, and K. Gallo, http://eprints.soton.ac.uk/42411/ 